\title{
Acute Heart Rate Responses to Resistance Exercise at Different Cadences
}

\author{
Marc R Apkarian* \\ Department of Kinesiology and Health Science, Biola University, USA
}

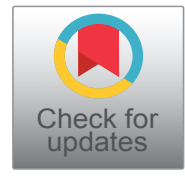

*Corresponding author: Marc R. Apkarian, Department of Kinesiology and Health Science, Biola University, 13800 Biola Avenue, La Mirada, CA 90639, USA, Tel: 562.944.0351 x5948, ORCID iD: 0000-0003-3700-3857

\begin{abstract}
Purpose: The purpose of this study was to evaluate whether movement speed, or cadence, affects the heart rate $(\mathrm{HR})$ response to three sets of 10 repetitions of resistance exercise at slow (6 seconds/repetition) and fast (2 seconds/repetition) cadences.

Methods: The 12 Repetition Maximum (12-RM) load on the angled leg press was determined for 16 moderately trained males $(26.8 \pm 6.2 \mathrm{yrs}, 177.7 \pm 6.3 \mathrm{~cm}, 77.0 \pm 10.9 \mathrm{~kg})$. To ensure safe, proper form across all sets, the 12-RM was tested at the slow cadence, and only 10 repetitions/set were performed during trials. Three successive sets of 10 repetitions, with three minutes rest between sets, were completed at each cadence, requiring 60 seconds to complete each slow set, 20 seconds to complete each fast set. Continuous $\mathrm{HR}$ was assessed by electrocardiogram from rest throughout exercise, and to recovery. Repeated-Measures (RM) ANOVA was applied, evaluating HR at each cadence for the exercises performed. Bonferroni adjustment resulted in a statistical significance of $p<0.017$.

Results: Peak HR (slow) was $112.8 \pm 12.2$ (set 1), $115.6 \pm$ 13.9 (set 2) and $119.1 \pm 17.1$ (set 3) bpm. Peak HR (fast) was $118.0 \pm 10.5$ (set 1), $116.0 \pm 10.1$ (set 2) and $116.8 \pm$ 10.2 (set 3) bpm. Significant change occurred for the increase in peak HR at the slow cadence only, with the fast cadence showing no increase, as both conditions demonstrate different response patterns.

Conclusion: When three sets of 10 repetitions are performed on the angled leg press at a slow cadence, a significant increase in peak HR occurs for the sets, while the fast cadence does not demonstrate an increase in peak HR values. The is presumed in part due to influences of the pressor response, and the longer work duration for the slow cadence of 60 seconds required per set of 10 repetitions, as compared with the shorter work duration of 20 seconds for 10 repetitions per set at the fast cadence.
\end{abstract}

Practical applications: Acute variability of the peak HR response to resistance exercise is possible through program modification. For a traditional resistance exercise arrangement such as three sets of 10 repetitions, it is possible to limit elevations in peak HR across sets by using short duration sets with fast repetitions, which may benefit populations for whom cardiovascular workload may be of concern.

\section{Keywords}

Hemodynamic, Sets, Repetitions, Angled leg press, 12-Repetition maximum

\section{Introduction}

Acute hemodynamic responses to exercise have long been investigated primarily under cardiorespiratory (aerobic) exercise conditions, owing to reasons such as its multiple conferred benefits, nature and format, and overall popularity [1]. Nevertheless, resistance exercise is also widely performed among the general public, and special populations, to enhance health status, physical conditioning, and human performance, presenting its own unique conditions and physiological demands of interest, including those on the cardiovascular system [2,3].

As a cardiovascular variable of fundamental significance, acute Heart Rate (HR) measures are seen to increase from rest to work at the onset of resistance exercise $[4,5]$. Traditional resistance exercise includes a combination of static and dynamic contractions, varying in proportion to the relative effort needed to lift and lower a load [4]. A static contraction of sufficient magnitude to overcome resistance of a load must oc-

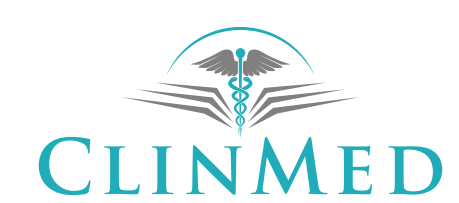

INTERNATIONAL LIBRARY 
cur, subsequent to which dynamic, concentric action maintains movement, followed by eccentric action to return the load to its starting point [6].

As such, hemodynamic changes having to do with resistance exercise reflect specific elements of its performance. Acute HR changes will be manifested as a function of many variables, including load, movement velocity (or cadence), rest interval length, amount of muscle mass involved, exercise duration, age, and training status $[4,5,7,8]$. These factors present widely varying physiological demands, causing HR to respond accordingly to the performance of repetitions and sets.

Since HR can be readily assessed via wired or wireless Electrocardiogram (ECG) units, or wireless heart rate monitors worn around the torso or a limb, acute $\mathrm{HR}$ responses can be monitored across a wide variety of resistance exercise modalities, and as a part of many resistance training studies. When movement velocity has been a variable of interest, investigations have often contrasted traditional, isotonic resistance exercise (performed as one to three sets of 10 repetitions at approximately $65-70 \%$ of the 1-Repetition Maximum, or $1 \mathrm{RM})$ with methods such as circuit weight training or super slow training [4,9-11].

Typically, circuit weight training occurs at a rapid pace with little to no rest in between sets performed for time (e.g. 60 seconds) on several multiple and/or single-joint exercises sequenced into a circuit, often repeated one or more times in exercise bouts lasting over 10 minutes $[4,10]$. In contrast, super slow training might involve one or more sets of eight repetitions of several multiple and/or single-joint exercises, using a 10 second concentric/ 5 second eccentric action movement velocity (15 seconds/repetition), resulting in 120 seconds/set for eight repetitions $[9,11]$.

By their nature and durations then, both circuit and super slow training systems necessitate the use of relatively light loads, equivalent to approximately $25-50 \%$ of the 1RM, being performed for 60 seconds or longer per set. These loads are comparatively lower than those used in most traditional resistance exercise training programs, where, for example, one set of 10 repetitions may last 30 seconds or less, with movement velocity usually disregarded. These distinctions among training systems have been proposed in previous studies as responsible for producing differing effects on acute HR responses, with slower velocities typically associated with attainment of lower peak HR values in a set or session than higher ones, irrespective of load assignments [911].

The aim of this study was to evaluate how cadence affects the acute $H R$ response during traditional resistance exercise, as further understanding about acute hemodynamic responses to resistance exercise can benefit specific program design goals, and applications within apparently healthy and special populations. To facilitate this objective, sets, repetitions, and load were held constant across different cadences while HR was measured continuously, and three sets of 10 repetitions were performed bilaterally on the angled leg press exercise, at both a slow cadence and a fast cadence, for a total of six sets.

\section{Methods}

\section{Subjects}

Eighteen healthy male volunteers aged 18-39 were recruited for participation in the investigation. Each participant provided informed consent in accordance with parameters approved by the institutional review board and completed a health history questionnaire prior to testing. Participants were excluded based on age criteria, present or past hypertension, significant cardiac or orthopedic disease, history of other disease that would significantly affect exercise testing, current or previous tobacco smoking, and unfamiliarity with resistance exercise. Subjects had to be at least moderately trained (exercising > $2.5 \mathrm{hrs} /$ week, with at least $0.5 \mathrm{hrs} /$ week moderate to intense exercise for the last three months) and had to be familiar with resistance exercise, but without any current or past competitive resistance training or weight lifting experience. Based upon the inclusion criteria, as well as eventual post-testing results, viable data from 16 subjects was processed and analyzed in total. Descriptive characteristics for all subjects are listed in Table 1.

\section{Experimental approach}

Due to the fact that the magnitude of hemodynamic changes to resistance exercise is influenced by specific exercise selection and modality, a multiple-joint exercise using a large absolute amount of muscle mass was expected to elicit the greatest distinction among acute HR responses $[5,7,8]$. In order to achieve this, the angled leg press exercise was selected to be performed in bilateral fashion, and as a machine with a fixed movement pattern, also enabled consistent technique and performance across subjects.

The acute HR response was measured continuously during each set of 10 repetitions, performed for three consecutive sets of 10 repetitions. Sets were performed both at a slow and at a fast cadence. Using a metronome for audible cues, the slow cadence was maintained at three seconds per concentric and eccentric phase (six seconds/repetition, or 10 repetitions/minute, totaling 60 seconds), and the fast cadence at one second per

Table 1: Descriptive data of subjects $(\underline{N}=16)$.

\begin{tabular}{|l|l|}
\hline Age (yrs) & $26.8 \pm 6.2$ \\
\hline Height (cm) & $177.7 \pm 6.3$ \\
\hline Weight (kg) & $77.0 \pm 10.9$ \\
\hline
\end{tabular}

Values are means \pm standard deviations. 
concentric and eccentric phase (two seconds/repetition, or 30 repetitions/minute, totaling 60 seconds). A 12-Repetition Maximum (12-RM) load was used during all trials to ensure the feasibility and safety of performing the six total sets of 10 repetitions during testing.

\section{Preliminary visit}

A preliminary visit to the laboratory was held to familiarize subjects with the experimental protocol and confirm via manual sphygmomanometry that resting values for Systolic Blood Pressure (SBP) were below $140 \mathrm{mmHg}$, and Diastolic Blood Pressure (DBP) values were below $90 \mathrm{mmHg}$. Subjects were directed to avoid stimulants such as caffeine, alcohol, and vigorous endurance exercise for 24 hours, and resistance exercise for 48 hours prior to the preliminary visit, as well as for the experimental session. Age, height, weight, resting heart rate and blood pressure were recorded. If the blood pressure for any subject was greater than 140/90 $\mathrm{mmHg}$, the subject was asked to remain seated quietly and motionless for 10 minutes before it was re-measured. If the second measurement was not acceptable, the subject was asked to return no sooner than 48 hours for the process to be repeated. If suitable results were not obtained during a second visit, subjects were not allowed to continue in the study.

The preliminary visit was also used to determine the 12-RM load value for the angled leg press. A 43-degree angled leg press (Body Masters, Rayne, LA) with a sled weight of 125 pounds was used in testing, however only weight plate values added on the sled were counted in determining loads, as the sled weight was fixed. Additionally, the 12-RM test was performed at the slow cadence due to its longer duration, as an "overshoot" feature in order to ensure that 10 repetitions could be performed safely for six total sets under both slow and fast cadences.

Once seated in the apparatus, each subject was positioned with the feet centered (both top to bottom and left to right) and placed hip-width on the foot plate of the angled leg press sled. Subjects were directed to maintain pressure evenly through the entire sole of the foot when lifting (pressing) or lowering the sled so that the heel or ball of the foot was not used exclusively. Upon beginning the exercise, subjects were instructed to lower the sled as far as that point at which a 90 bend was created at the knee. Similarly, when pressing the sled, they were asked to achieve a $180^{\circ}$ angle at the knee, without locking the knee. Subjects were coached to avoid performing a Valsalva maneuver, being instructed to exhale while pressing the sled, and inhale while lowering it. Repetitions were counted audibly for the subjects during exercise.

Before determination of the 12-RM load, a warmup set of 15 repetitions on the angled leg press was completed with a load approximately equal to half the subject's body weight. Subjects were instructed to use a comfortable, self-selected speed during the warm-up exercise set. Subsequent to this set, the load was increased to double that of the warm-up (or more, based upon subject reported information) in an attempt to determine the 12-RM load. At this point, subjects were instructed to follow the metronome-based cadence of three seconds per lifting and lowering movement. If a subject was able to complete 12 repetitions such that the last repetition did not produce momentary muscular failure, the load was increased by approximately 20$40 \%$ of body weight during subsequent attempts until the 12-RM load was determined. In cases where additional weight did not permit 12 repetitions, $10-20 \%$ of body weight was subtracted from the load and another attempt was made. A three-minute rest period was provided between all attempts. The testing protocol and rest periods used during this session were adapted from protocols reported elsewhere [12].

\section{Experimental session}

After a minimum of 48 hours but not more than 10 days, subjects returned for the experimental session. The experimental session began with one warm-up set of 15 repetitions using the same load and a self-selected speed, as with the preliminary visit. This was followed by three sets of 10 repetitions with the 12-RM load at both the slow cadence, and at the fast cadence. The order in which the three sets were performed at either cadence (slow then fast, or fast then slow) was alternated with each subject.

Following each of the three sets performed at either cadence during the experimental session, a three-minute rest period was allowed. To ensure consistency in procedures, as well as general safety and recovery, at least 10 minutes between the entire three sets of exercise at either cadence was permitted for reductions in $\mathrm{HR}$, as well as SBP and DBP to within $5 \mathrm{mmHg}$ of resting values. If any blood pressure did not return to within 5 $\mathrm{mmHg}$ of its resting value within 45 minutes, subjects were not allowed to continue participation.

Furthermore, flexion and extension movements of the knee were recorded using an electronic goniometer (TSD 180, BIOPAC Systems, Goleta, CA) that was calibrated during the subject set-up period through the use of a manual goniometer. This was performed so that entire repetitions, as well as their concentric and eccentric phases, could be identified easily during subsequent analyses.

\section{Heart rate assessment}

Continuous, beat-by-beat recording of heart rate values was performed with a typical three lead electrocardiogram (ECG) configuration using leads I, II, III. Analog signals of ECG waveforms were derived through an automated device (Colin Pilot $^{\circledR}$, Colin Medical Instruments Corporation, San Antonio, Texas), and electronically in- 
Table 2: Peak heart rate values by set and cadence.

\begin{tabular}{|l|l|l|}
\hline Trial & HR slow (bpm) & HR fast (bpm) \\
\hline Rest & $72.8 \pm 13.1$ & $68.4 \pm 9.0$ \\
\hline Peak Set 1 & $112.8 \pm 12.2$ & $118.0 \pm 10.5$ \\
\hline Post Set 1 & $81.4 \pm 16.3$ & $70.9 \pm 12.1$ \\
\hline Peak Set 2 & $115.6 \pm 13.9$ & $116.0 \pm 10.1$ \\
\hline Post Set 2 & $84.4 \pm 19.8$ & $74.1 \pm 14.0$ \\
\hline Peak Set 3 & $119.1 \pm 17.1^{*}$ & $116.8 \pm 10.2$ \\
\hline Post Set 3 & $87.0 \pm 19.7$ & $75.0 \pm 13.4$ \\
\hline
\end{tabular}

Values are means \pm standard deviations; *Significantly different than Peak Set 1 ( $\underline{0}<0.017)$.

Table 3: Reference data for rest and recovery blood pressure.

\begin{tabular}{|l|l|l|}
\hline \multicolumn{2}{|c|}{ Manual Sphygmomanometry } \\
\hline Trial & SBP $(\mathbf{m m H g})$ & DBP $(\mathbf{m m H g})$ \\
\hline Slow & & \\
\hline Rest & $112.5 \pm 7.8$ & $66.3 \pm 7.1$ \\
\hline Post Set 1 & $126.1 \pm 11.5$ & $64.4 \pm 7.1$ \\
\hline Post Set 2 & $128.5 \pm 10.9$ & $63.5 \pm 8.0$ \\
\hline Post Set 3 & $132.3 \pm 10.9$ & $62.6 \pm 9.8$ \\
\hline Fast & & \\
\hline Rest & $112.5 \pm 6.5$ & $63.8 \pm 7.8$ \\
\hline Post Set 1 & $125.0 \pm 10.8$ & $67.0 \pm 7.0$ \\
\hline Post Set 2 & $125.8 \pm 9.1$ & $68.4 \pm 7.1$ \\
\hline Post Set 3 & $127.9 \pm 9.8$ & $69.0 \pm 7.3$ \\
\hline
\end{tabular}

Values are means \pm standard deviations.

tegrated via data acquisition hardware (MP 100, BIOPAC systems, Goleta, CA).

\section{Data processing}

Raw signal input was sampled at a frequency of 500 $\mathrm{Hz}$ for joint angle and heart rate. Data was initially acquired as voltages; however heart rate values were subsequently converted to beats per minute (bpm). Joint angle voltages did not require conversion, as calibration of the electronic goniometer allowed for on-screen tracings that indicated flexion and extension of the knee between $90^{\circ}$ and $180^{\circ}$ as peaks and nadirs on the graphical output.

For heart rates, a 20 second average of data was determined at rest starting 60 seconds prior to the beginning of set 1 of both the slow and fast trials. Additionally, 20 second heart rate averages were determined starting 60 seconds after the completion of the final repetition of any set. The 20 second averaging intervals and start times were used to coincide with start times and approximate duration of manual sphygmomanometry measurements, used to monitor general safety in rest and recovery periods.

Due to the nature of the angled leg press mechanism of performance, repetitions commenced by lowering the sled before lifting it, resulting in an eccentric action prior to a concentric one. These phases of a rep- etition were identified by time using goniometer signal tracings. Thus, one complete repetition was counted from the start of an eccentric action to the completion of a concentric action. Values for heart rate by repetition were counted as the final beat of any given repetition.

\section{Statistical procedures}

For analysis of peak heart rates, repeated measures (RM) ANOVA was applied for repetitions and sets for each of the cadences (slow and fast). Additionally, the procedure was applied to peak heart rate for cadence, repetitions, and sets.

In the absence of interaction, main effects were tested using simple main effects analyses. Statistical significance was initially accepted at a level of $p<0.05$. Bonferroni adjustment resulted in a final statistical significance level of $p<0.017(0.05 / 3)$. Statistica software vs. 10.0 (Statsoft, Tulsa, OK, USA) was used in data analysis.

\section{Results}

Rest, peak, and recovery heart rate values for trials by set and cadence appear in Table 2. Across sets, peak $\mathrm{HR}$ demonstrated a significant increase at the slow cadence, but not at the fast cadence $(p<0.017)$. Table 3 presents reference data for rest and recovery blood pressure values. Representative tracings of raw data for joint angle and ECG tracings are illustrated in Figure 1.

Significant difference was noted for the trend, or pattern of results, of the main effects of peak HR values for the slow vs. fast cadences across the three sets of exercise $(p<0.017)$. Figure 2 illustrates the increase of peak $H R$ across sets at the slow, but not fast, cadence.

Figure 3 presents graphical summaries of mean HR data by cadence and repetition for three consecutive sets. For clarity, rest, recovery and inter-set data is omitted, and error bars of the first and last repetitions of a set are presented.

\section{Discussion}

The findings of this study are supported by previous observations that cardiovascular demand increases in proportion to exercise intensity and duration. This stems from increased muscle blood flow and active hyperemia during resistance exercise, primarily from direct motor unit and accessory muscle recruitment needed for exercise, and as fatigue occurs, resulting in acute HR elevations $[5,13]$.

A relatively linear increasing trend exists for HR values across repetitions at the slow and fast cadences as shown in Figure 3, which is similar to most cardiovascular exercise bouts of incrementally increasing intensity. In this study, peak HR of any set at either cadence never exceeded $120 \mathrm{bpm}$. However, another leg press resistance exercise study yielded acute HR values as high as $186 \mathrm{bpm}$ in five repetitions of the leg press when performed with a 5-RM load [14]. Such differences suggest 

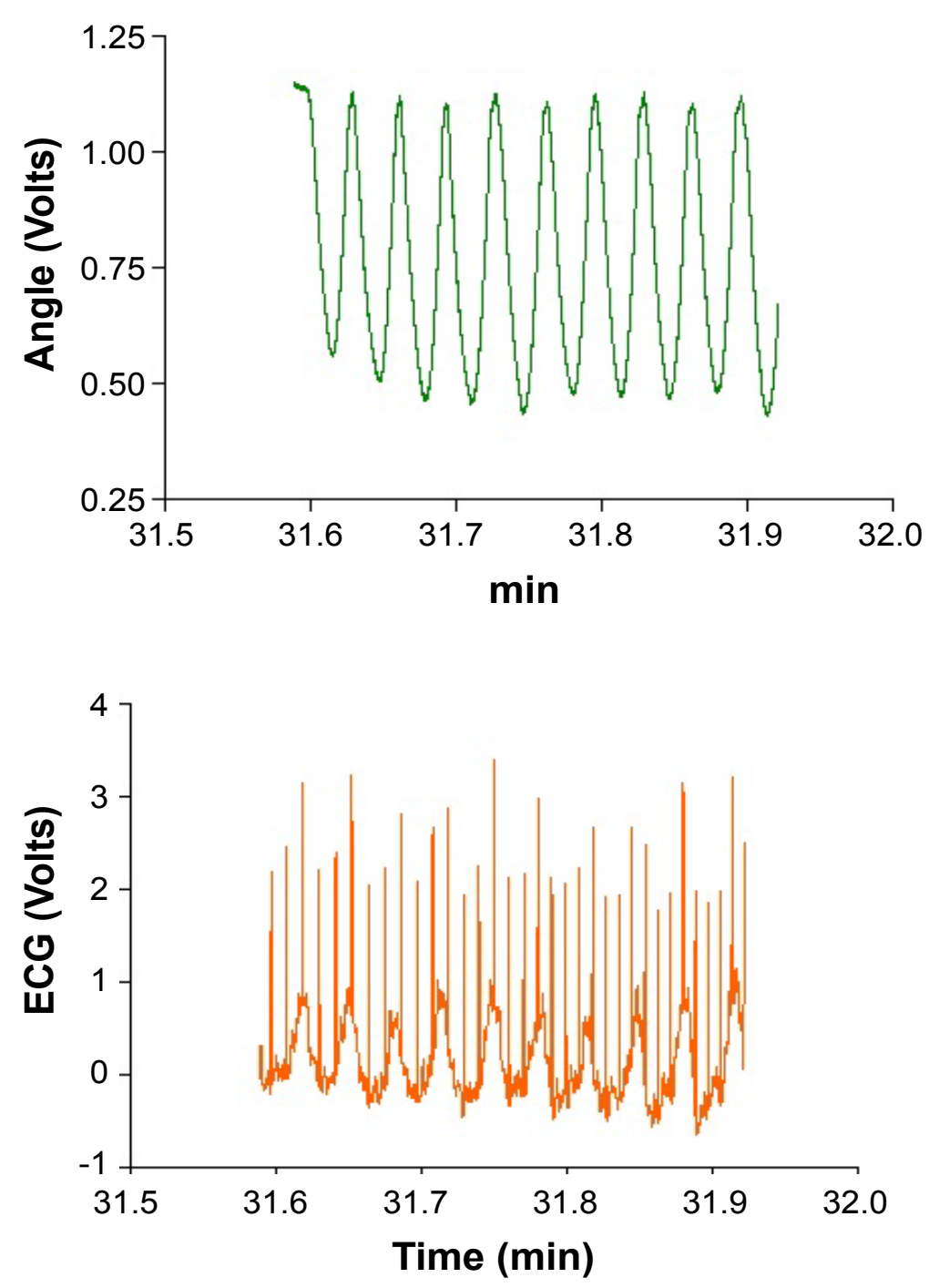

Figure 1: Raw data sample for knee joint angle and ECG tracing from a subject.

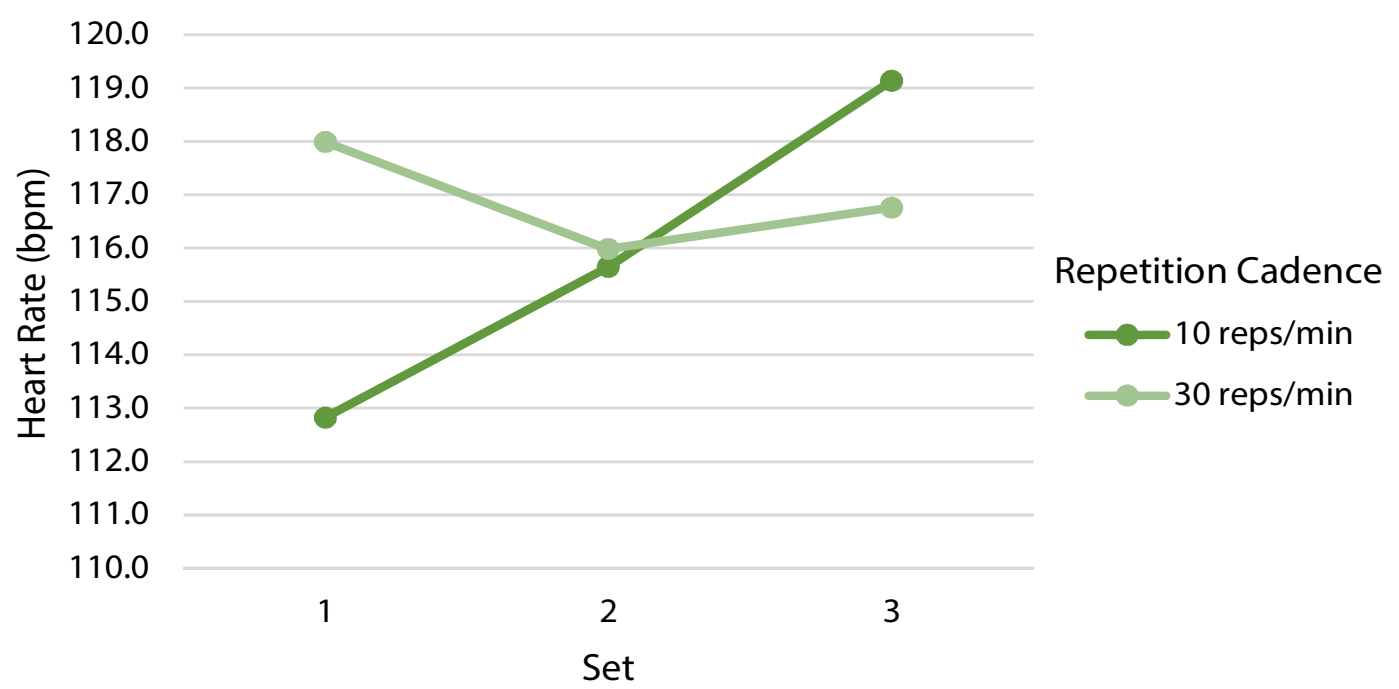

Figure 2: Trends for peak HR values by cadence.

a proportionate nature for peak HR achieved during resistance exercise, relative to the absolute loads used.

Across three sets, a significant increase in peak HR was observed at the slow, but not the fast cadence. This is presumably due in part to an immediate and exaggerated pressor response resulting from the condition, as well as longer work duration of 60 seconds for the slow 


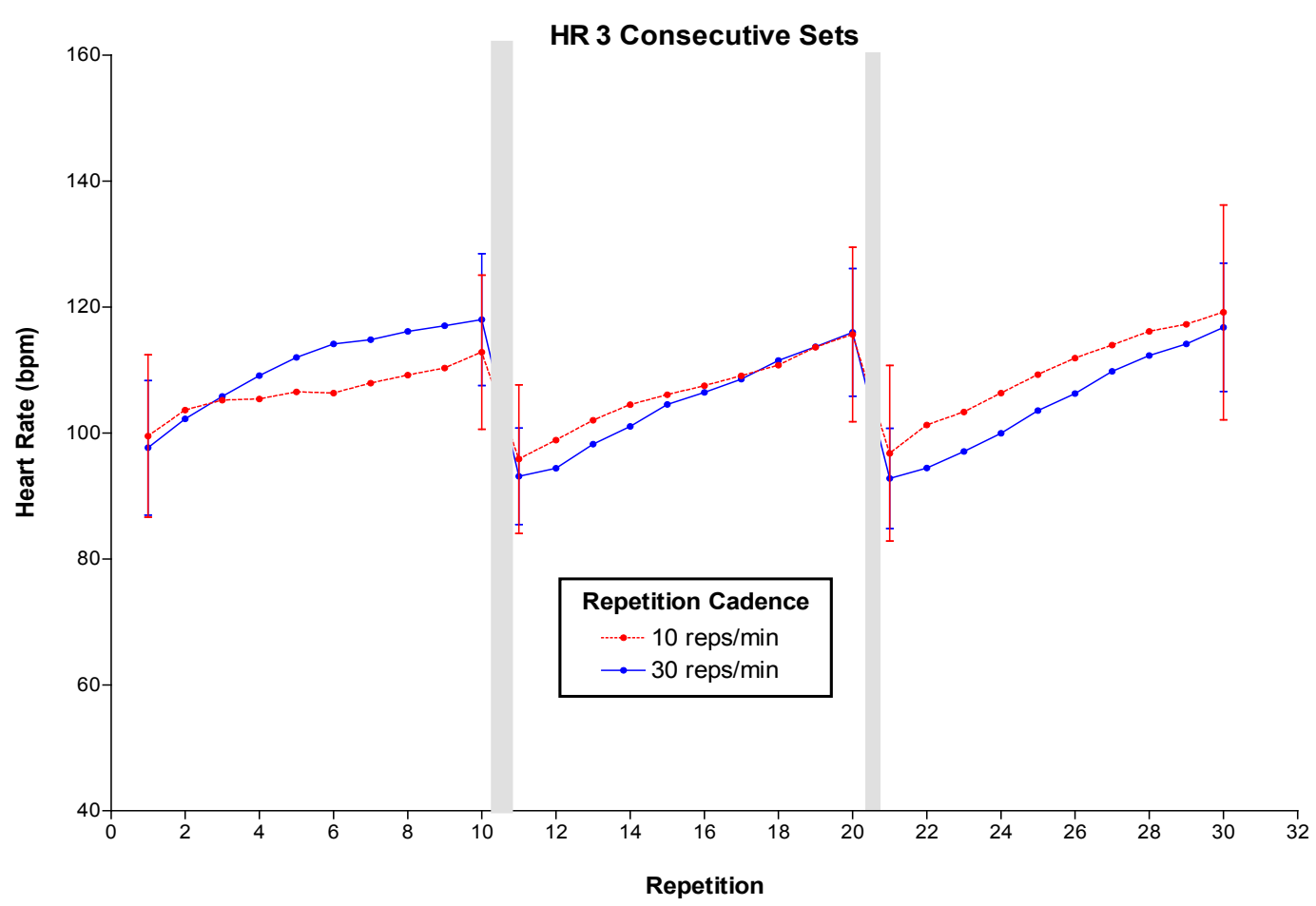

Figure 3: Mean values for heart rate by repetition across 3 sets of 10 repetitions.

trials, as compared with 20 seconds for the fast trials $[14,15]$. The longer duration of the slow sets requires greater total work and creates more metabolic demand, likely influencing HR to remain elevated more so than for the fast sets. Moreover, as the three-minute rest period was identical for both conditions, it is proportionately longer for the fast trials (180 seconds rest: 20 seconds work), than the slow trials ( 180 seconds rest: 60 seconds work). It is also likely that extending the recovery period would be necessary from set to set at the slow cadence to allow additional lowering of HR values post exercise, similar to the profile of the fast cadence, as in Table 2.

Additionally, the significant difference noted for main effects of peak HR values for the slow vs. fast cadences across three sets is evident in the trend pattern of results obtained, as seen in Figure 2. However, this indicates there is no similarity existing in the pattern of change between results obtained under the two conditions. For the peak HR values, as indicated earlier, no peak exceeded $120 \mathrm{bpm}$, which represents only a relatively modest cardiovascular intensity for the subject population. Also, as shown in Table 3, the 60 second recovery HR, SBP, and DBP values following all sets also remained under $90 \mathrm{bpm}$ and $140 / 90 \mathrm{mmHg}$, reflecting a lower intensity was attained for these variables prior to performance of subsequent sets of exercise.

In considering influences on HR values during resistance exercise, modality and posture may impact hemodynamics of movements performed in alternative fashions. For instance, the seated leg press, used in several leg press studies, requires an upright seated position with legs outstretched approximately at hip level, differing from the angled leg press, where the exerciser is seated at ground level with feet placed on the sled above heart level $[6,14,16-18]$. In the angled leg press, venous return to the heart may be augmented posture and the effect of gravity, possibly creating stroke volume and vasoconstrictive changes as well, which may blunt the extent to which acute HR elevations may occur, as compared with the seated leg press.

Another aspect of exercise performance that can affect HR responses is load assignment and voluntary exertion. In this study, 10 repetitions were performed using a 12-RM load tested at the slow cadence to ensure the safety and feasibility of performing the six total sets in the experimental session. This load, however, did not induce complete muscular failure at the end of each set, and since the degree to which subjects voluntarily exerted themselves cannot be known, outcomes must be viewed accordingly. As such, it is possible the 10 repetitions performed with the $12-\mathrm{RM}$ load did not produce as high of peak HR values as a heavier (i.e. 10RM) load might have, and actual momentary muscular failure been achieved.

Finally, while invoking the Valsalva maneuver is known to increase blood pressures, it can influence heart rate as well. In one resistance training study involving breathing technique, Narloch and Brandstater demonstrated that combining the Valsalva maneuver with performance of the leg press using a 5-RM load yielded a maximum blood pressure in one subject of $370 / 360 \mathrm{mmHg}$, and $\mathrm{HR}$ of $186 \mathrm{bpm}$, while breathing slowly for the same effort produced a pressure of 220/200 mmHg and HR of 132 bpm [14]. At extreme levels of exertion, it has been reported that the Valsalva maneuver is almost obligatory [16]. Though it was not 
possible to control, each subject in the present study was instructed how to breathe so as to avoid performing a Valsalva maneuver. While performance of 10 repetitions at a 12-RM load on the angled leg press would not be expected to elicit extreme hemodynamic responses associated with maximal efforts and the Valsalva maneuver, any extent to which it may have had and influence is unclear.

In understanding the role of cadence in this study, it is useful to contrast the traditional resistance exercise model with the forms of circuit weight training, and of super-slow training. In a study by Pichon, et al., subjects completed four exercises of a circuit weight training protocol using two sets of 20 repetitions (at $\sim 47 \%$ of $1-\mathrm{RM}$ ) for 60 seconds per set, with 30 seconds rest between sets [10]. The same exercises were performed in traditional fashion, using two sets of 10 repetitions (at $\sim 69 \%$ of $1-\mathrm{RM}$ ) for 30 seconds per set, with 90 seconds rest between sets. Although the relative load used was greater, HR values (averaged throughout exercise and recovery across each condition) achieved during traditional resistance exercise were $120 \mathrm{bpm}$, as compared with the circuit, at $135 \mathrm{bpm}$, despite the fact that the resulting cadence was the same under both conditions (three seconds/repetition). It was concluded that the higher result obtained from circuit training was attributed to its longer work and shorter rest periods than that of the traditional method, despite the lower loads used in the circuit.

In two investigations comparing the super slow model of resistance exercise with traditional resistance exercise, higher HR values were observed in the traditional models $[9,11]$. An investigation by Hunter, et al. used 10 exercises in a super slow training method of one set of 8 repetitions, at a cadence of $10 \mathrm{sec}-$ onds/concentric action, five seconds/eccentric action, and the same exercises in a traditional method of two sets of 8 repetitions, at a cadence of one second for each concentric and eccentric action [9]. Total exercise time, inclusive of rest periods, was held constant at 29 minutes/session for both methods, with subjects using $25 \%$ of $1-\mathrm{RM}$ values during super slow training, and $65 \%$ of $1-\mathrm{RM}$ values during traditional training. During the work period of super slow sessions, averaged HR values were found to be $113 \mathrm{bpm}$, while during traditional training they reached $143 \mathrm{bpm}$.

In a separate investigation comparing super slow and traditional resistance exercise, Wickwire, et al. demonstrated a similar outcome, whereby super slow training yielded lower peak HR values than traditional resistance exercise when comparing three sets each of knee extension and elbow flexion exercises [11]. This super slow method used 40\% 1-RM loads, and a cadence of 10 seconds/concentric action and five seconds/eccentric action, while the traditional method relied upon 65\% 1-RM loads, and a cadence of two seconds/concentric action and four seconds/eccentric action. Both conditions required repetitions to be performed to failure, with no target value specified. Across both exercises and all three sets, traditional resistance exercise elicited higher peak HR values (no peak above $145 \mathrm{bpm}$ ) than did super slow training. These results were attributed to the higher loads and faster cadence of the traditional method, which by its nature also facilitated performance of more repetitions per set than during super slow training.

Across these three studies, the general trend observed in the attainment of higher HR values during various forms of resistance exercise tends to parallel those conditions with a relatively faster cadence when compared with those of a slower cadence, notwithstanding the load assignments used [9-11]. Stated differently, these studies showed that circuit training yielded higher HR values when compared with traditional resistance exercise, and traditional resistance exercise elicited higher HR values when contrasted with super slow training.

This investigation, employing a different - that is, traditional resistance exercise - design than the aforementioned circuit and super slow studies, demonstrates some similarity to those findings, in that the peak HR values for the slow cadence exhibited the trend they did, as compared with the fast cadence. It is almost certain that the longer duration and additive effect of the greater physical effort needed for three successive sets performed under the slow condition creates increased physiological burden on the cardiovascular system. This is also plausible based on the fact that the absolute load used under both conditions was identical, while the total time to perform each set (60 seconds slow vs. 20 seconds fast) differed.

The results of this study likely reflect the physiological responses of common resistance exercise, as three sets of 10 repetitions (with two to six second per repetition cadences) are common parameters in general fitness practice. This assumes use of traditional resistance exercise models, with loads producing failure or fatigue by the end of the set. Furthermore, these results suggest that for a constant repetition target value, a faster cadence, with a shorter set duration, may reduce the extent to which HR is elevated, and minimize cardiovascular strain. This may be of specific benefit to populations such as cardiac patients, those with cardiovascular health risks, or others who must restrict their participation in activities which may otherwise result in sustained hypertensive states.

Additionally, limiting the number of sets of a specific exercise might also reduce the possibility for additive exercise intensity from set to set that substantially elevates HR. Also, diversifying the type (e.g. upper and lower body) or number of muscle groups exercised within an exercise session, often occurring in popular practice, 
can help divert blood flow from one region to another, possibly allowing enhanced localized and systemic hemodynamic recovery. For the angled leg press in particular, its user position and effect on cardiovascular dynamics may also contribute to reduced $H R$ values attained during exercise, suggesting the same effect may be similarly experienced in other seated or non-standing exercise positions.

Future opportunities for investigation include the use of repetitions, sets, loads, rest periods, and modalities other than those of this study, along with a greater number and variety of subjects tested. In additional research, conversion of HR values to represent percentage increases, or examining basal vs. peak values, may also reduce naturally occurring variabilities, perhaps making significant changes more visible. In regard to cadence, various resistance training approaches also lend themselves to additional avenues of study. One such example could include assessing acute HR and other hemodynamic responses to the same exercise, but with varying intensities such as 6-, 8-, 10-, 12-, or 15-RM loads. Another approach might involve evaluating such responses to equal (e.g. 1 second concentric phase, 1 second eccentric phase, common in general fitness) and variable (e.g. $3 \mathrm{sec}-$ onds concentric phase, 5 seconds eccentric phase, as in hypertrophy periodization) repetition cadences, which is not very clearly defined at present. Finally, the multiple possibilities for investigation of exercise arrangements with mixtures of traditional resistance exercise, circuit weight training, super slow training, Olympic lifting, or other combinations of isotonic, isometric, and/or isokinetic exercise might yield new, novel insights to various hemodynamic characteristics and responses.

\section{Acknowledgments}

Grateful appreciation is given to Robert A. Robergs, Ph.D., as well as to the Exercise Physiology Laboratories of the Department of Health, Exercise and Sports Sciences at the University of New Mexico, where data collection occurred.

\section{References}

1. Garber CE, Blissmer B, Deschenes MR, Franklin BA, Lamonte MJ, et al. (2011) American College of Sports Medicine position stand. Quantity and quality of exercise for developing and maintaining cardiorespiratory, musculoskeletal, and neuromotor fitness in apparently healthy adults: guidance for prescribing exercise. Med Sci Sports Exerc 43: 13341359.

2. Piercy KL, Troiano RP, Ballard RM, Carlson SA, Fulton JE, et al. (2018) The Physical Activity Guidelines for Americans. JAMA 320: 2020-2028.

3. Williams MA, Haskell WL, Ades PA, Amsterdam EA, Bittner V, et al. (2007) Resistance Exercise in Individuals with and Without Cardiovascular Disease: 2007 Update : a scientific statement from the American Heart Association Council on Clinical Cardiology and Council on Nutrition, Physical Activ- ity, and Metabolism. Circulation 116: 572-584.

4. Hill DW, Butler SD (1991) Hemodynamic responses to weightlifting exercise. Sports Med 12: 1-7.

5. McCartney N (1999) Acute responses to resistance training and safety. Med Sci Sports Exerc 31: 31-37.

6. MacDougall JD, Tuxen D, Sale DG, Moroz JR, Sutton JR (1985) Arterial blood pressure response to heavy resistance exercise. J Appl Physiol (1985) 58: 785-790.

7. de Vos NJ, Singh NA, Ross DA, Stavrinos TM, Orr R, et al. (2008) Continuous hemodynamic response to maximal dynamic strength testing in older adults. Arch Phys Med Rehabil 89: 343-350.

8. Moreira OC, Faraci LL, de Matos DG, Mazini Filho ML, da Silva SF, et al. (2017) Cardiovascular Responses to Unilateral, Bilateral, and Alternating Limb Resistance Exercise Performed Using Different Body Segments. J Strength Cond Res 31: 644-652.

9. Hunter GR, Seelhorst D, Snyder S (2003) Comparison of metabolic and heart rate responses to super slow vs. traditional resistance training. J Strength Cond Res 17: 76-81.

10. Pichon CE, Hunter GR, Morris M, Bond RL, Metz J (1996) Blood Pressure and Heart Rate Response and Metabolic Cost of Circuit Versus Traditional Weight Training. J Strength Cond Res 10: 153-156.

11. Wickwire PJ, McLester JR, Green JM, Crews TR (2009) Acute Heart Rate, Blood Pressure, and RPE Responses During Super Slow vs. Traditional Machine Resistance Training Protocols Using Small Muscle Group Exercises. J Strength Cond Res 23: 72-79.

12. National Strength and Conditioning Association (2000) Resistance Training. In: Baechle TR, Earle RW, Essentials of Strength Training and Conditioning. ( $2^{\text {nd }}$ edn), Champaign, IL: Human Kinetics, 452-457.

13. Mayo JJ, Kravitz LK (1999) A review of the acute cardiovascular responses to resistance exercise of healthy young and older adults. J Strength Cond Res 13: 90-96.

14. Narloch JA, Brandstater ME (1995) Influence of breathing technique on arterial blood pressure during heavy weight lifting. Arch Phys Med Rehabil 76: 457-462.

15. Fleck SJ, Dean LS (1987) Resistance-training experience and the pressor response during resistance exercise. $J$ Appl Physiol 63: 116-120.

16. MacDougall JD, McKelvie RS, Moroz DE, Sale DG, McCartney N, et al. (1992) Factors affecting blood pressure during heavy weight lifting and static contractions. J Appl Physiol 73: $1590-1597$.

17. McCartney N, McKelvie RS, Martin J, Sale DG, MacDougall JD (1985) Weight-training-induced attenuation of the circulatory response of older males to weight lifting. J Appl Physiol 74: 1056-1060.

18. Sale DG, Moroz DE, McKelvie RS, MacDougall JD, McCartney $N$ (1994) Effect of training on the blood pressure response to weight lifting. Can J Appl Physiol 19: 60-74. 